\title{
One-pot synthesis of pyrano[3,2-c]pyran derivatives catalyzed by $\mathrm{KF} / \mathrm{Al}_{2} \mathrm{O}_{3}$
}

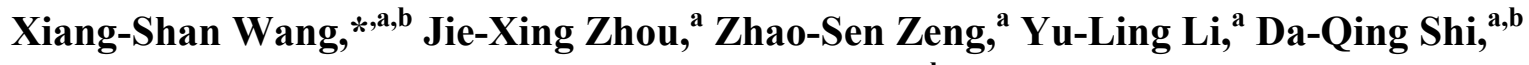 \\ and Shu-Jiang $T u^{\mathrm{a}, \mathrm{b}}$ \\ ${ }^{a}$ Department of Chemistry, Xuzhou Normal University, Xuzhou Jiangsu, 221116, China \\ ${ }^{b}$ The Key Laboratory of Biotechnology for Medical Plant, Jiangsu, Xuzhou 221116, China \\ E-mail: xswang1974@yahoo.com
}

\begin{abstract}
A series of pyrano[3,2-c]pyran derivatives have been synthesized by the reaction of aromatic aldehyde, malononitrile or cyanoacetate and 4-hydroxy-5-methylpyran-2-one in EtOH at room temperature catalyzed by $\mathrm{KF} / \mathrm{Al}_{2} \mathrm{O}_{3}$. The structures of the products were characterized by IR, ${ }^{1} \mathrm{H}$ NMR and elemental analysis, and $4 \mathrm{a}$ was further confirmed by X-ray diffraction analysis.
\end{abstract}

Keywords: Pyrano[3,2-c]pyran, $\mathrm{KF} / \mathrm{Al}_{2} \mathrm{O}_{3}$, synthesis

\section{Introduction}

It is known that many pyran derivatives exhibit a wide spectrum of pharmacological activities and biological activities at melanocortin receptors being used in the design of peptidomimetics relating to a tripeptide structure, ${ }^{1}$ such as fungicidal, insecticidal and acaricidal activity, ${ }^{2}$ antiviral activity, ${ }^{3}$ miticidal activity, ${ }^{4}$ stimulant activity, ${ }^{5}$ and anticonvulsant activity. ${ }^{6}$ These promoted us to synthesis these compounds via a new way. Particularly, we focused our attention on the use of KF-alumina as catalyst, because the utility of fluoride salts as potential base in a variety of synthetic reactions has been recognized in recent years, ${ }^{7}$ resulting in their higher selectivity, milder reaction conditions and easier work-up. Especially alumina coated with potassium fluoride (KF-alumina) has been a versatile solid-supported reagent developed by Ando et al. for alkylation. ${ }^{8}$ Over the years the reagent has been found application in a large number of organic reactions. ${ }^{9}$ In order to further enlarge the application of the reagent of KF-alumina, in this paper, we would like to report one pot synthesis these potential active pyrano[3,2-c]pyran derivatives by the reaction of aromatic aldehyde, malononitrile or cyanoacetate and 4-hydroxy-6methylpyran-2-one catalyzed by KF-alumina at room temperature. 


\section{Results and Discussion}

When aromatic aldehyde (1), malononitrile, or cyanoacetate (2) and 4-hydroxy-6-methylpyran-2one (3) were treated with $\mathrm{KF}-\mathrm{Al}_{2} \mathrm{O}_{3}$ in ethyl alcohol at room temperature, 2-amino-4-aryl-4H, $5 H$-pyrano[3,2-c]pyran-5-one derivatives (4) were obtained in slightly high yields (75-98\%) (Scheme 1).<smiles>[R]C[C@H](C)[C@@H](C)O[CH]</smiles>

Scheme 1

Table 1. The reaction time and the yields of the products 4

\begin{tabular}{ccccc}
\hline Entry & $\mathrm{Ar}$ & $\mathrm{R}$ & Time(h) & Yields (\%) \\
\hline $\mathbf{4 a}$ & $3-\mathrm{NO}_{2} \mathrm{C}_{6} \mathrm{H}_{4}$ & $\mathrm{CN}$ & 6 & 75 \\
$\mathbf{4 b}$ & $4-\mathrm{BrC}_{6} \mathrm{H}_{4}$ & $\mathrm{CN}$ & 8 & 94 \\
$\mathbf{4 c}$ & $2-\mathrm{ClC}_{6} \mathrm{H}_{4}$ & $\mathrm{CN}$ & 8 & 98 \\
$\mathbf{4 d}$ & $4-\mathrm{ClC}_{6} \mathrm{H}_{4}$ & $\mathrm{CN}$ & 8 & 76 \\
$\mathbf{4 e}$ & $4-\mathrm{Cl}_{2}-\mathrm{NO}_{2} \mathrm{C}_{6} \mathrm{H}_{3}$ & $\mathrm{CN}$ & 5 & 84 \\
$\mathbf{4} \mathbf{f}$ & $3,4-\mathrm{Cl}_{2} \mathrm{C}_{6} \mathrm{H}_{3}$ & $\mathrm{CN}$ & 5 & 83 \\
$\mathbf{4 g}$ & $2,4-\mathrm{Cl}_{2} \mathrm{C}_{6} \mathrm{H}_{3}$ & $\mathrm{CN}$ & 5 & 86 \\
$\mathbf{4 h}$ & $3-\mathrm{ClC}_{6} \mathrm{H}_{4}$ & $\mathrm{CN}$ & 8 & 94 \\
$\mathbf{4 i}$ & $3,4-\mathrm{Cl}_{2} \mathrm{C}_{6} \mathrm{H}_{3}$ & $\mathrm{CO}_{2} \mathrm{Et}$ & 8 & 93 \\
$\mathbf{4 j}$ & $2,4-\mathrm{Cl}_{2} \mathrm{C}_{6} \mathrm{H}_{3}$ & $\mathrm{CO}_{2} \mathrm{Et}$ & 8 & 87 \\
$\mathbf{4} \mathbf{k}$ & $3-\mathrm{ClC}_{6} \mathrm{H}_{4}$ & $\mathrm{CO}_{2} \mathrm{Et}$ & 10 & 82 \\
$\mathbf{4 \mathbf { l }}$ & $2-\mathrm{ClC}_{6} \mathrm{H}_{4}$ & $\mathrm{CO}_{2} \mathrm{Et}$ & 10 & 81 \\
$\mathbf{4 m}$ & $4-\mathrm{ClC}_{6} \mathrm{H}_{4}$ & $\mathrm{CO}_{2} \mathrm{Et}$ & 10 & 94 \\
$\mathbf{4 n}$ & $3,4-\mathrm{Cl}_{2} \mathrm{C}_{6} \mathrm{H}_{3}$ & $\mathrm{CO}_{2} \mathrm{Me}$ & 8 & 88 \\
\hline
\end{tabular}

In order to demonstrate the efficiency and the applicability of the present method, we performed the reaction of a variety of aromatic aldehyde with malononitrile or cyanoacetate and 3 in EtOH at room temperature and in the presence of $\mathrm{KF}-\mathrm{Al}_{2} \mathrm{O}_{3}$. As shown in Table 1, we can see a series of $\mathbf{1}$ reacted with $\mathbf{2}$ and $\mathbf{3}$ to give the corresponding products $\mathbf{4}$ in good yields under same reaction conditions.

The isolated pyrano[3,2-c]pyran derivatives 4 were completely characterized by $\mathrm{IR},{ }^{1} \mathrm{H}$ NMR and elemental analyses. The analyses were in agreement with their structures. The melting 
points of known compounds were conformed to those of the references reported. The IR spectra for $4 \mathbf{a}$ exhibited sharp bands at 3400, $3327 \mathrm{~cm}^{-1}\left(\mathrm{NH}_{2}\right), 2199 \mathrm{~cm}^{-1}(\mathrm{CN}), 1716 \mathrm{~cm}^{-1}(\mathrm{C}=\mathrm{O})$. The ${ }^{1} \mathrm{H}$ NMR spectrum of $4 \mathbf{a}$ exhibited a singlet identified as methyl (2.24), two singlets exhibited at 4.57 and $6.32 \mathrm{ppm}$ identified as two methines $(\mathrm{CH}$ and $\mathrm{CH}=)$, respectively, and along with multiplets (7.64-8.14) for aromatic protons. The $\mathrm{NH}$ proton resonance at 7.35 disappeared after addition of $\mathrm{D}_{2} \mathrm{O}$ to the DMSO- $d_{6}$ solution of $4 \mathbf{a}$. In order to further confirm the structure of the product, the X-ray analysis ${ }^{10}$ of $\mathbf{4 a}$ was carried out. The crystal structure of $\mathbf{4 a}$ was shown in Figure 1.

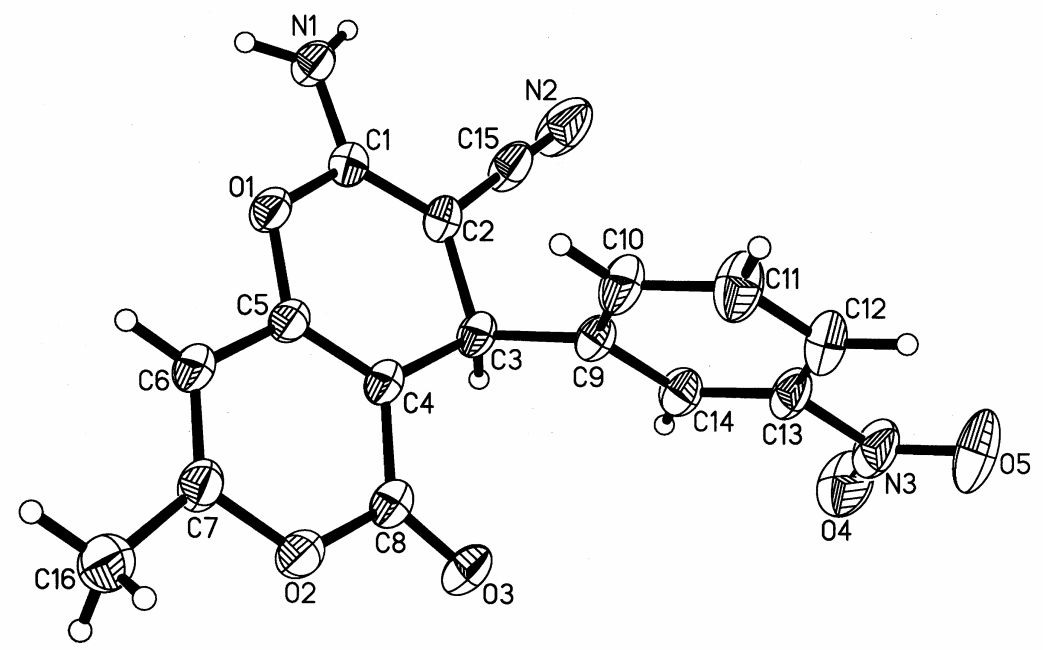

Figure 1. The crystal structure of $4 \mathbf{a}$.

Although the detailed mechanism of the above reaction has not been clarified yet, the formation of $\mathbf{4}$ can be explained by the possible mechanism presented in Scheme 2.

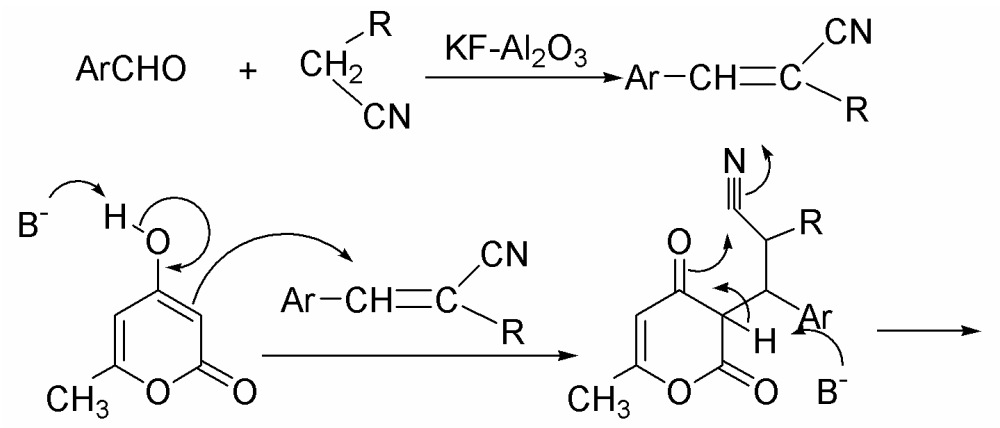

\section{Scheme 2}<smiles>[R]C1=C(N)Oc2cc(C)oc(=O)c2C1[Al]</smiles> 
In conclusion, we find a novel one-pot method available for the synthesis of pyrano[3,2c]pyran derivatives. Meanwhile, the new method also further expands the application of the catalyst of $\mathrm{KF}-\mathrm{Al}_{2} \mathrm{O}_{3}$ in organic synthesis. Compared with other methods, ${ }^{11}$ this method has the advantage of one-step, easy work-up, milder reaction conditions and good yields in synthesis these potential active compounds.

\section{Experimental Section}

General Procedures. Melting points were determined in open capillaries and are uncorrected. IR spectra were recorded on a TENSOR 27 spectrometer in KBr. ${ }^{1} \mathrm{H}$ NMR spectra were obtained for solutions in DMSO- $d_{6}$ with $\mathrm{Me}_{4} \mathrm{Si}$ as internal standard using a Bruker-400 spectrometer. Elemental analyses were carried out using Carlo Erba 1110 analyzer. X-ray diffraction was measured on a Siemens P4 diffractometer.

General procedure for pyrano[3,2-c]pyran derivatives (4). A dry $50 \mathrm{~mL}$ flask was charged with aromatic aldehyde $\mathbf{1}(2 \mathrm{mmol})$, malononitrile or cyanoacetate $\mathbf{2}(2.5 \mathrm{mmol})$ and 4-hydroxy6-methylpyran-2-one 3 (2 mmol), $\mathrm{KF}-\mathrm{Al}_{2} \mathrm{O}_{3}(100 \mathrm{mg})$ and ethyl alcohol $(10 \mathrm{~mL})$, the mixture was stirred at room temperature for 5-10 h. The mixture was poured into $200 \mathrm{~mL}$ water, the solid filtered off, and washed with water. The crude product was purified by recrystallization from DMF and water to give 4.

2-Amino-3-cyano-4-(3-nitrophenyl)-4H,5H-pyrano[3,2-c]pyran-5-one (4a). Pale yellow crystals; m.p. $238-240{ }^{\circ} \mathrm{C}$ (lit. ${ }^{11} 234-235^{\circ} \mathrm{C}$ ). ${ }^{1} \mathrm{H}$ NMR (DMSO- $d_{6}, \delta$, ppm) $\delta: 2.24$ (s, 3H, $\mathrm{CH}_{3}$ ), $4.57(\mathrm{~s}, 1 \mathrm{H}, \mathrm{CH}), 6.32(\mathrm{~s}, 1 \mathrm{H},=\mathrm{CH}), 7.35\left(\mathrm{~s}, 2 \mathrm{H}, \mathrm{NH}_{2}\right), 7.64(\mathrm{t}, J=8.0 \mathrm{~Hz}, 1 \mathrm{H}, \mathrm{ArH}), 7.73(\mathrm{tt}, J=$ $\left.8.0 \mathrm{~Hz}, J^{\prime}=1.2 \mathrm{~Hz}, 1 \mathrm{H}, \mathrm{ArH}\right), 8.05$ (t, $\left.J=2.0 \mathrm{~Hz}, 1 \mathrm{H}, \mathrm{ArH}\right), 8.13(\mathrm{dd}, \mathrm{dd}, J=8.0 \mathrm{~Hz}, J=2.0$ $\left.\mathrm{Hz}, J^{\prime}=1.2 \mathrm{~Hz}, 1 \mathrm{H}, \mathrm{ArH}\right) . \mathrm{IR}\left(\mathrm{KBr}, \mathrm{cm}^{-1}\right): 3400,3327,2199,1716,1615,1526,1448,1383$, $1263,1200,1143,1024,977,817,759,733 \mathrm{~cm}^{-1}$.

2-Amino-3-cyano-4-(4-bromophenyl)-4H,5H-pyrano[3,2-c]pyran-5-one (4b). Pale yellow crystals; m.p. $223-225{ }^{\circ} \mathrm{C}$ (lit. ${ }^{11} 223-224{ }^{\circ} \mathrm{C}$ ). ${ }^{1} \mathrm{H}$ NMR (DMSO-d $d_{6}, \delta$, ppm): $2.23\left(\mathrm{~s}, 3 \mathrm{H}, \mathrm{CH}_{3}\right.$ ), $4.31(\mathrm{~s}, 1 \mathrm{H}, \mathrm{CH}), 6.29(\mathrm{~s}, 1 \mathrm{H},=\mathrm{CH}), 7.17(\mathrm{~d}, 2 \mathrm{H}, J=8.4 \mathrm{~Hz}, \mathrm{ArH}), 7.26\left(\mathrm{~s}, 2 \mathrm{H}, \mathrm{NH}_{2}\right), 7.51$ (d, $2 \mathrm{H}, J=8.4 \mathrm{~Hz}, \mathrm{ArH}) . \mathrm{IR}\left(\mathrm{KBr}, \mathrm{cm}^{-1}\right): 3388,3324,2201,1707,1644,1589,1486,1445,1408$, $1314,1261,1196,1176,1142,1012,982,854,829,776$.

2-Amino-3-cyano-4-(2-chlorophenyl)-4H,5H-pyrano[3,2-c]pyran-5-one (4c). Pale yellow crystals; m.p. $270-271{ }^{\circ} \mathrm{C}$ (lit. ${ }^{11} 267-268{ }^{\circ} \mathrm{C}$ ). ${ }^{1} \mathrm{H}$ NMR (DMSO- $d_{6}, \delta$, ppm): $2.24\left(\mathrm{~s}, 3 \mathrm{H}, \mathrm{CH}_{3}\right)$, $4.79(\mathrm{~s}, 1 \mathrm{H}, \mathrm{CH}), 6.29(\mathrm{~s}, 1 \mathrm{H},=\mathrm{CH}), 7.21-7.32\left(\mathrm{~m}, 5 \mathrm{H}, \mathrm{ArH}+\mathrm{NH}_{2}\right), 7.39(\mathrm{dd}, 1 \mathrm{H}, J=7.6 \mathrm{~Hz}, J$ $=1.2 \mathrm{~Hz}, \mathrm{ArH}) . \mathrm{IR}\left(\mathrm{KBr}, \mathrm{cm}^{-1}\right): 3471,3344,3104,2192,1700,1638,1579,1473,1443,1377$, 1263, 1197, 1180, 1139, 1043, 964, 820, 762.

2-Amino-3-cyano-4-(4-chlorophenyl)-4H,5H-pyrano[3,2-c]pyran-5-one (4d). Pale yellow crystals; m.p. $231-232{ }^{\circ} \mathrm{C}$ (lit. ${ }^{11} 230-231{ }^{\circ} \mathrm{C}$ ). ${ }^{1} \mathrm{H}$ NMR (DMSO- $d_{6}, \delta$, ppm): $2.23\left(\mathrm{~s}, 3 \mathrm{H}, \mathrm{CH}_{3}\right.$ ), $4.32(\mathrm{~s}, 1 \mathrm{H}, \mathrm{CH}), 6.29(\mathrm{~s}, 1 \mathrm{H},=\mathrm{CH}), 7.23(\mathrm{~d}, 2 \mathrm{H}, J=8.4 \mathrm{~Hz}, \mathrm{ArH}), 7.26\left(\mathrm{~s}, 2 \mathrm{H}, \mathrm{NH}_{2}\right), 7.37$ (d, 
$2 \mathrm{H}, J=8.4 \mathrm{~Hz}, \mathrm{ArH}) . \mathrm{IR}\left(\mathrm{KBr}, \mathrm{cm}^{-1}\right): 3382,3324,2202,1711,1645,1590,1488,1445,1414$, 1385, 1314, 1261, 1196, 1092, 1015, 981, 854, 830, 807, 777.

2-Amino-3-cyano-4-(4-chloro-2-nitrophenyl)-4H,5H-pyrano[3,2-c]pyran-5-one (4e). Pale yellow crystals; m.p. $247-249{ }^{\circ} \mathrm{C} .{ }^{1} \mathrm{H}$ NMR (DMSO- $d_{6}, \delta$, ppm): 2.09 (s, 3H, CH ), 5.08 (s, 1H, $\mathrm{CH}), 6.30(\mathrm{~s}, 1 \mathrm{H},=\mathrm{CH}), 7.44\left(\mathrm{~s}, 2 \mathrm{H}, \mathrm{NH}_{2}\right), 7.54(\mathrm{~d}, 1 \mathrm{H}, J=2.0 \mathrm{~Hz}, \mathrm{ArH}), 7.59$ (dd, $1 \mathrm{H}, J=8.8$ $\left.\mathrm{Hz}, J^{\prime}=2.0 \mathrm{~Hz}, \mathrm{ArH}\right), 7.93(\mathrm{~d}, 1 \mathrm{H}, J=8.8 \mathrm{~Hz}, \mathrm{ArH}) . \mathrm{IR}\left(\mathrm{KBr}, \mathrm{cm}^{-1}\right): 3419,3332,2195,1703$, 1644, 1588, 1522, 1449, 1382, 1338, 1260, 1198, 1145, 1040, 977, 900, 849, 199, 774. Anal. calcd for $\mathrm{C}_{16} \mathrm{H}_{10} \mathrm{ClN}_{3} \mathrm{O}_{5}$ : C 53.42, H 2.80, N 11.68. Found: C 53.29, H 2.91, N 11.52.

2-Amino-3-cyano-4-(3,4-dichlorophenyl)-4H,5H-pyrano[3,2-c]pyran-5-one (4f). Pale yellow crystals; m.p. $239-241{ }^{\circ} \mathrm{C} .{ }^{1} \mathrm{H}$ NMR (DMSO- $d_{6}, \delta$, ppm): $2.23\left(\mathrm{~s}, 3 \mathrm{H}, \mathrm{CH}_{3}\right), 4.39(\mathrm{~s}, 1 \mathrm{H}, \mathrm{CH})$, $6.29(\mathrm{~s}, 1 \mathrm{H},=\mathrm{CH}), 7.22(\mathrm{dd}, 1 \mathrm{H}, J=8.0 \mathrm{~Hz}, J=2.0 \mathrm{~Hz}, \mathrm{ArH}), 7.32\left(\mathrm{~s}, 2 \mathrm{H}, \mathrm{NH}_{2}\right), 7.49(\mathrm{~d}, 1 \mathrm{H}, J$ $=2.0 \mathrm{~Hz}, \mathrm{ArH}), 7.58(\mathrm{~d}, 1 \mathrm{H}, J=8.0 \mathrm{~Hz}, \mathrm{ArH}) . \mathrm{IR}\left(\mathrm{KBr}, \mathrm{cm}^{-1}\right): 3391,3326,2200,1715,1645$, 1590, 1465, 1445, 1381, 1298, 1261, 1185, 1141, 1031, 983, 969, 898, 810, 786, 770. Anal. calcd for $\mathrm{C}_{16} \mathrm{H}_{10} \mathrm{Cl}_{2} \mathrm{~N}_{2} \mathrm{O}_{3}$ : C 55.04, H 2.89, N 8.02. Found: C 54.90, H 2.99, $\mathrm{N} 8.00$.

2-Amino-3-cyano-4-(2,4-dichlorophenyl)-4H,5H-pyrano[3,2-c]pyran-5-one (4g). Pale yellow crystals; m.p. $234-235^{\circ} \mathrm{C}$ (lit. ${ }^{11} 230-231{ }^{\circ} \mathrm{C}$ ). ${ }^{1} \mathrm{H}$ NMR (DMSO- $d_{6}, \delta$, ppm): 2.24 (s, 3H, $\mathrm{CH}_{3}$ ), 4.79 (s, 1H, CH), 6.30 (s, 1H, =CH), 7.29 (d, 1H, J=8.0 Hz, ArH), 7.30 (s, 2H, NH $), 7.38$ (dd, $1 \mathrm{H}, J=8.0 \mathrm{~Hz}, J=2.0 \mathrm{~Hz}, \mathrm{ArH}), 7.57(\mathrm{~d}, 1 \mathrm{H}, J=2.0 \mathrm{~Hz}, \mathrm{ArH}) . \mathrm{IR}\left(\mathrm{KBr}, \mathrm{cm}^{-1}\right): 3408,3348$, 2196, 1706, 1642, 1583, 1472, 1444, 1376, 1265, 1198, 1137, 1105, 1047, 966, 857, 828, 738.

2-Amino-3-cyano-4-(3-chlorophenyl)-4H,5H-pyrano[3,2-c]pyran-5-one (4h). Pale yellow crystals; m.p. $255-257{ }^{\circ} \mathrm{C} .{ }^{1} \mathrm{H}$ NMR (DMSO- $d_{6}, \delta$ ppm): $2.24\left(\mathrm{~s}, 3 \mathrm{H}, \mathrm{CH}_{3}\right), 4.35$ (s, $\left.1 \mathrm{H}, \mathrm{CH}\right)$, $6.29(\mathrm{~s}, 1 \mathrm{H},=\mathrm{CH}), 7.17(\mathrm{~d}, 1 \mathrm{H}, J=7.6 \mathrm{~Hz}, \mathrm{ArH}), 7.25-7.38\left(\mathrm{~m}, 5 \mathrm{H}, \mathrm{ArH}+\mathrm{NH}_{2}\right) . \mathrm{IR}(\mathrm{KBr}, v$, $\left.\mathrm{cm}^{-1}\right): 3396,3323,2198,1707,1644,1590,1473,1442,1430,1383,1303,1259,1179,1140$, 1041, 980, 833, 803, 770. Anal. calcd for $\mathrm{C}_{16} \mathrm{H}_{11} \mathrm{ClN}_{2} \mathrm{O}_{3}$ : C 61.06, H 3.52, N 8.90. Found: C 61.04, H 3.70, N 8.77.

Ethyl 2-amino-3-cyano-4-(3,4-dichlorophenyl)-5-oxo-4H,5H-pyrano[3,2-c]pyran-3- carboxylate (4i). Pale yellow crystals; m.p. $179-181{ }^{\circ} \mathrm{C}$. IR (KBr, $\left.v, \mathrm{~cm}^{-1}\right): 3447,3320,1983,1717$, 1690, 1622, 1513, 1468, 1448, 1378, 1286, 1257, 1211, 1174, 1138, 1086, 1030, 976, 814, 787; ${ }^{1} \mathrm{H}$ NMR (DMSO- $d_{6}, \delta$, ppm): $1.08\left(\mathrm{t}, 3 \mathrm{H}, J=6.8 \mathrm{~Hz}, \mathrm{CH}_{3}\right), 2.10\left(\mathrm{~s}, 3 \mathrm{H}, \mathrm{CH}_{3}\right), 3.97$ (q, $2 \mathrm{H}, J=$ $\left.6.8 \mathrm{~Hz}, \mathrm{CH}_{3}\right), 4.52(\mathrm{~s}, 1 \mathrm{H}, \mathrm{CH}), 6.32(\mathrm{~s}, 1 \mathrm{H},=\mathrm{CH}), 7.15(\mathrm{dd}, 1 \mathrm{H}, J=8.8 \mathrm{~Hz}, J=2.0 \mathrm{~Hz}, \mathrm{ArH})$, $7.38(\mathrm{~d}, 1 \mathrm{H}, J=2.0 \mathrm{~Hz}, \mathrm{ArH}), 7.52(\mathrm{~d}, 1 \mathrm{H}, J=8.8 \mathrm{~Hz}, \mathrm{ArH}) 7.82\left(\mathrm{~s}, 2 \mathrm{H}, \mathrm{NH}_{2}\right)$. Anal. calcd for $\mathrm{C}_{18} \mathrm{H}_{12} \mathrm{Cl}_{2} \mathrm{NO}_{5}$ : C 54.56, H 3.82, N 3.54. Found: C 54.48, H 3.93, N 3.50.

Ethyl 2-amino-3-cyano-4-(2,4-dichlorophenyl)-5-oxo-4H,5H-pyrano[3,2-c]pyran-3- carboxylate (4j). Pale yellow crystals; m.p. 206-208 ${ }^{\circ} \mathrm{C}$ (lit. ${ }^{11} 204-205{ }^{\circ} \mathrm{C}$ ). ${ }^{1} \mathrm{H}$ NMR (DMSO- $d_{6}, \delta$, ppm): 1.04 (t, 3H, J=7.2 Hz, $\left.\mathrm{CH}_{3}\right), 2.10\left(\mathrm{~s}, 3 \mathrm{H}, \mathrm{CH}_{3}\right), 3.92$ (q, 2H, J=7.2 Hz, $\left.\mathrm{CH}_{3}\right), 4.88$ (s, $1 \mathrm{H}, \mathrm{CH}), 6.29(\mathrm{~s}, 1 \mathrm{H},=\mathrm{CH}), 7.26(\mathrm{~d}, 1 \mathrm{H}, J=8.4 \mathrm{~Hz}, \mathrm{ArH}), 7.32(\mathrm{dd}, 1 \mathrm{H}, J=8.4 \mathrm{~Hz}, \mathrm{~J}$ ' = 2.4 Hz, $\operatorname{ArH}), 7.43$ (d, $1 \mathrm{H}, J=2.4 \mathrm{~Hz}, \mathrm{ArH}), 7.85$ (s, 2H, $\left.\mathrm{NH}_{2}\right)$. IR $\left(\mathrm{KBr}, \mathrm{cm}^{-1}\right): 3435,3298,2957,1712$, 1689, 1615, 1584, 1505, 1471, 1380, 1290, 1250, 1174, 1139, 1080, 1046, 957, 849, 817.

Ethyl 2-amino-3-cyano-4-(3-chlorophenyl)-5-oxo-4H,5H-pyrano[3,2-c]pyran-3-carboxylate (4k). Pale yellow crystals; m.p. $180-182{ }^{\circ} \mathrm{C} .{ }^{1} \mathrm{H}$ NMR (DMSO- $d_{6}, \delta$, ppm): 1.08 (t, $3 \mathrm{H}, J=6.8$ 
$\left.\mathrm{Hz}, \mathrm{CH}_{3}\right), 2.10\left(\mathrm{~s}, 3 \mathrm{H}, \mathrm{CH}_{3}\right), 3.96\left(\mathrm{q}, 2 \mathrm{H}, J=6.8 \mathrm{~Hz}, \mathrm{CH}_{3}\right), 4.52(\mathrm{~s}, 1 \mathrm{H}, \mathrm{CH}), 6.32(\mathrm{~s}, 1 \mathrm{H},=\mathrm{CH})$, 7.17 (d, 1H, $J=7.6 \mathrm{~Hz}, \mathrm{ArH}), 7.11-7.31$ (m, 4H, ArH), 7.79 (s, 2H, NH$)_{2}$. IR $\left(\mathrm{KBr}, \mathrm{cm}^{-1}\right): 3426$, 3294, 2979, 1710, 1687, 1619, 1506, 1475, 1442, 1378, 1286, 1252, 1212, 1174, 1140, 1073, 1041, 958, 816, 782, 750. Anal. calcd for $\mathrm{C}_{18} \mathrm{H}_{16} \mathrm{ClNO}_{5}$ : C 59.76, H 4.46, N 3.87. Found: C 59.76, H 4.57, N 3.72 .

Ethyl 2-amino-3-cyano-4-(2-chlorophenyl)-5-oxo-4H,5H-pyrano[3,2-c]pyran-3-carboxylate (4I). Pale yellow crystals; m.p. 203-204 ${ }^{\circ} \mathrm{C}$ (lit. ${ }^{11} 203-205{ }^{\circ} \mathrm{C}$ ). ${ }^{1} \mathrm{H}$ NMR (DMSO- $d_{6}, \delta$, ppm): $1.03\left(\mathrm{t}, 3 \mathrm{H}, J=7.2 \mathrm{~Hz}, \mathrm{CH}_{3}\right), 2.22\left(\mathrm{~s}, 3 \mathrm{H}, \mathrm{CH}_{3}\right), 3.92\left(\mathrm{q}, 2 \mathrm{H}, J=7.2 \mathrm{~Hz}, \mathrm{CH}_{3}\right), 4.90(\mathrm{~s}, 1 \mathrm{H}, \mathrm{CH})$, $6.29(\mathrm{~s}, 1 \mathrm{H},=\mathrm{CH}), 7.14-7.24(\mathrm{~m}, 3 \mathrm{H}, \mathrm{ArH}), 7.29(\mathrm{~d}, 1 \mathrm{H}, J=8.0 \mathrm{~Hz}, \mathrm{ArH}), 7.81\left(\mathrm{~s}, 2 \mathrm{H}, \mathrm{NH}_{2}\right)$. IR $\left(\mathrm{KBr}, \mathrm{cm}^{-1}\right): 3421,3295,2988,1715,1688,1621,1509,1440,1379,1287,1176,1077,980,956$, $815,773,756$.

Ethyl 2-amino-3-cyano-4-(4-chlorophenyl)-5-oxo-4H,5H-pyrano[3,2-c]pyran-3-carboxylate (4m). Pale yellow crystals; m.p. $160-161{ }^{\circ} \mathrm{C}$ (lit. ${ }^{11} 156-157{ }^{\circ} \mathrm{C}$ ). ${ }^{1} \mathrm{H}$ NMR (DMSO- $d_{6}, \delta$, ppm): $1.07\left(\mathrm{t}, 3 \mathrm{H}, J=6.8 \mathrm{~Hz}, \mathrm{CH}_{3}\right), 2.22\left(\mathrm{~s}, 3 \mathrm{H}, \mathrm{CH}_{3}\right), 3.95$ (q, 2H, $\left.J=6.8 \mathrm{~Hz}, \mathrm{CH}_{3}\right), 4.52(\mathrm{~s}, 1 \mathrm{H}, \mathrm{CH})$, $6.31(\mathrm{~s}, 1 \mathrm{H},=\mathrm{CH}), 7.19(\mathrm{~d}, 2 \mathrm{H}, J=8.4 \mathrm{~Hz}, \mathrm{ArH}), 7.30(\mathrm{~d}, 2 \mathrm{H}, J=8.4 \mathrm{~Hz}, \mathrm{ArH}), 7.77$ (s, 2H, $\mathrm{NH}_{2}$ ). IR (KBr, cm $\left.{ }^{-1}\right): 3431,3306,2980,1715,1678,1618,1490,1443,1378,1291,1215,1172$, 1140, 1076, 1014, 981, 955, 841, 818 .

Methyl 2-amino-3-cyano-4-(3,4-dichlorophenyl)-5-oxo-4H,5H-pyrano[3,2-c]pyran- 3carboxylate (4n). Pale yellow crystals; m.p. 193-197 ${ }^{\circ} \mathrm{C} .{ }^{1} \mathrm{H}$ NMR (DMSO- $\left.d_{6}, \delta, \mathrm{ppm}\right): 2.10$ (s, $\left.3 \mathrm{H}, \mathrm{CH}_{3}\right), 3.52\left(\mathrm{~s}, 3 \mathrm{H}, \mathrm{CH}_{3} \mathrm{O}\right), 4.53(\mathrm{~s}, 1 \mathrm{H}, \mathrm{CH}), 6.32(\mathrm{~s}, 1 \mathrm{H},=\mathrm{CH}), 7.17(\mathrm{dd}, 1 \mathrm{H}, J=8.4 \mathrm{~Hz}, J$ $=2.0 \mathrm{~Hz}, \mathrm{ArH}), 7.36(\mathrm{~d}, 1 \mathrm{H}, J=2.0 \mathrm{~Hz}, \mathrm{ArH}), 7.52(\mathrm{~d}, 1 \mathrm{H}, J=8.4 \mathrm{~Hz}, \mathrm{ArH}), 7.83\left(\mathrm{~s}, 2 \mathrm{H}, \mathrm{NH}_{2}\right)$. IR $\left(\mathrm{KBr}, \mathrm{cm}^{-1}\right): 3426,3304,2955,1712,1687,1619,1514,1468,1439,1380,1296,1254,1219$, 1176, 1141, 1085, 1033, 973, 941, 810, 774, 746. Anal. calcd for $\mathrm{C}_{17} \mathrm{H}_{13} \mathrm{Cl}_{2} \mathrm{NO}_{5}$ : C 53.42, $\mathrm{H}$ 3.43, N 3.66. Found: C 53.29, H 3.50, N 3.51.

\section{Supplementary Information Available}

Crystallographic data for the structure $\mathbf{4 a}$ reported in this paper has been deposited at the Cambridge Crystallographic Data Centre as supplementary publication with No. CCDC-605772. Copies of available material can be obtained, free of charge, on application to the Director, CCDC, 12 Union Road, Cambridge CB2 1EZ, UK, (fax: +44-(0) 1223-336033 or e-mail: deposit@ccdc.cam.ac.uk).

\section{Acknowledgements}

We are grateful to the Natural Science Foundation (04KJB150139) of the Education Committee of Jiangsu Province for financial support. 


\section{References and Footnotes}

1. Kulesza, A.; Ebetino, F. H.; Mishra, R. K.; Cross-Doersen, D.; Mazur, A. W. Organic Lett. 2003, $5,1163$.

2. Uher, M.; Konecny, V.; Rajniakove, O. Chem. Pap. 1994, 48, 282.

3. Perez-Perez, M.; Balzarini, J.; Rozenski, J.; De-Clercq, E.; Herdewijn, P. Bioorg. Med. Chem. Lett. 1995, 5, 1115.

4. Takao, H.; Murai, K.; Yasudomi, N.; Goto, T.; Umetsu, N.; Horie, T. Nippon Noyaku Gakkaishi, 1994, 19, 151.

5. Zamocka, J.; Misikova, E.; Durinda, J. Pharmazie, 1991, 46, 610.

6. Aytemir, M. D.; Calis, U.; Ozalp, M. Arch. Pharm., 2004, 337, 281.

7. Clark, J. H. Chem. Rev. 1980, 80, 429.

8. Ando, T.; Yamawaki, J.; Kawate, T.; Sumi, S.; Hanafusa, T. Bull. Chem. Soc. Jpn. 1982, 55, 2504.

9. (a) Villemin, D. J. Chem. Soc., Chem. Commun. 1985, 870. (b) Gao, Y.; Shi, D. Q.; Zhou, L. H.; Dai, G. Y. Chin. J. Org. Chem. 1996, 16, 548. (c) Melot, J. M.; Boullet, F. M.; Foucaud, A. Tetrahedron Lett. 1986, 27, 493. (d) Yamawaki, J.; Kawate, T.; Ando, T.; Hanafusa, T. Bull. Chem. Soc. Jpn. 1983, 56, 1885. (e) Boullet, F. M.; Villemin, D.; Ricard, M.; Moison, A.; Foucaud, A. Tetrahedron 1985, 41, 1259. (f) Wang, X. S.; Shi, D. Q.; Tu, S. J. Synth. Commun. 2003, 33, 119. (g) Wang, X. S.; Shi D. Q.; Yu, H. Z.; Wang, G. F.; Tu, S. J. Synth. Commun. 2004, 34, 509. (h) Wang, X. S.; Zeng, Z. S.; Shi, D. Q.; Wei, X. Y.; Zong, Z. M. Synth. Commun. 2004, 34, 3027.

10. X-ray crystallography for 4a: Empirical formula $\mathrm{C}_{16} \mathrm{H}_{11} \mathrm{~N}_{3} \mathrm{O}_{5}, F_{W}=325.28, T=289(2) \mathrm{K}$, Monoclinic, space group P2(1)/c, $a=5.709(1) \AA, b=14.616(2) \AA, c=18.228(3) \AA, \beta=$ $94.219(2)^{\circ}, V=1516.8(8) \AA^{3}, Z=4, D c=1.424 \mathrm{Mg} / \mathrm{m}^{3}, \lambda(M o K \alpha)=0.71073 \AA, \mu=0.109$ $\mathrm{mm}^{-1}, F(000)=672.1 .79^{\circ}<\theta<25.49^{\circ}, R=0.0423, w R=0.0961 . S=0.952$, Largest diff. Peak and hole: 0.153 and -0.181 e. $\AA^{-3}$

11. (a) Shi, D. Q.; Niu, L. H.; Wang, X. S.; Zhuang, Q. Y.; Zhang, Y. J. Chem. Res. (S) 2005, 724. (b) Shestopalov, A. M.; Zlotkin, S. G.; Shestopalov, A. A.; Mortikov, V. Y.; Rodinovskaya, L. A. Russ. Chem. Bull. 2004, 53, 573. (c) Shi, D. Q.; Niu, L. H.; Wang, X. S.; Zhuang, Q. Y.; Zhang, Y. Acta Cryst. 2004, E60, 1995. 